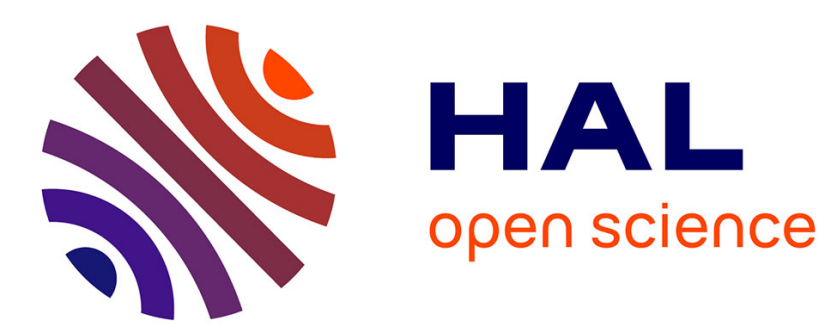

\title{
Caractérisation de l'aptitude à la congélation du sperme de truite arc-en-ciel (Salmo gairdneri) par des critères physico-chimiques
}

Gérard Maisse, A. Pinson, Maurice Loir

\section{- To cite this version:}

Gérard Maisse, A. Pinson, Maurice Loir. Caractérisation de l'aptitude à la congélation du sperme de truite arc-en-ciel (Salmo gairdneri) par des critères physico-chimiques. Aquatic Living Resources, 1988, 1, pp.45-51. 10.1051/alr:1988005 . hal-02728794

\section{HAL Id: hal-02728794 \\ https://hal.inrae.fr/hal-02728794}

Submitted on 2 Jun 2020

HAL is a multi-disciplinary open access archive for the deposit and dissemination of scientific research documents, whether they are published or not. The documents may come from teaching and research institutions in France or abroad, or from public or private research centers.
L'archive ouverte pluridisciplinaire HAL, est destinée au dépôt et à la diffusion de documents scientifiques de niveau recherche, publiés ou non, émanant des établissements d'enseignement et de recherche français ou étrangers, des laboratoires publics ou privés. 


\title{
Caractérisation de l'aptitude à la congélation du sperme de truite arc-en-ciel (Salmo gairdneri) par des critères physico-chimiques
}

\author{
Gérard Maisse, Nlain Pinson et Maurice Loir \\ INRA, Laboratoire de Physiologie des Poissons \\ Campus de Beaulieu, 35072 Rennes Cedex (France).
}

Reçu le 19 août 1987 , accepté le 24 novembre 1987.

Characterization of the fitness for cryopresertation of milt from rainbow trout (Salmo gairdneri) together with physico-chemical criteria.

Maisse G., A. Pinson, M. Loir, Aquat. Liting Resour., 1988, 1, 45-51.

Abstract

Milt was obtained from 40 males of a spring-spawning strain of rainbow trout (Salmo gairdneri). Only semen with a volume greater or equal to $6 \mathrm{ml}$ were characterized and frozen in pellets. Factorial analysis of correspondences between the physico-chemical characteristics and the fertility of sperm after 5 to 9 months freezing revealed that the fitness for cryoprescrvation can be particularly characterized by the composition of the seminal plasma: sperm with the lowest capacity to fertilize were associated with a plasma osmolality greater than $260 \mathrm{~m}-\mathrm{Osm}$ and/or a high level of $42 \mathrm{KD}$ protein, one of the major components of the spermatozoan membrane; sperm with the highest fertilizing ability lacked $42 \mathrm{KD}$ protein in the seminal plasma. These results support the idea that the fitness for cryopreservation of trout sperm primarily depends on the condition of the spermatozoan membrane.

Keywords : Trout, sperm, cryopreservation, seminal plasma.

Résumé

Un lot de 40 mâles d'une souche de truite arc-en-ciel (Salmo gairdneri) à reproduction printanière a été suivi. Seuls les spermes d'un volume supérieur ou égal à $6 \mathrm{ml}$ ont été caractérisés et congelés en boulettes. L'analyse factorielle des correspondances entre les caractéristiques et la fécondance du sperme après 5 à 9 mois de congélation a montré en particulier que l'aptitude à la cryoconservation peut être caractérisée par l'analyse du plasma séminal : les spermes médiocres sont associés à une osmolarité plasmatique élevće ( $260 \mathrm{~m}$-osmoles) et/ou une teneur ćlevée en protéine $42 \mathrm{KD}$, l'un des constituants majeur de la membrane des spermatozoïdes; les très bons spermes sont associés à l'absence de protéine $42 \mathrm{KD}$ dans le liquide séminal. Ces résultats renforcent l'idée que l'aptitude à la cryoconservation du sperme de truite dépend en premier lieu de la qualité de la membrane des spermatozoïdes.

Mots-clés : Truite, sperme, congèlation, plasma séminal.

\section{INTRODUCTION}

La cryoconservation du sperme de salmonidé a fait l'objet de nombreuses ćtudes (revues bibliographiques de Scott et Baynes, 1980 et de Stoss, 1983) qui ont abouti à la mise au point de différents dilueurs de congćlation aux performances comparables (Stoss et Holtz cités par Stoss et Donaldson, 1982). Ott et Horton (1971) ont mis en évidence des diffẻrences significatives de taux de fécondation après congélation entre mâles de truite tête d'acier (Salmo gairdneri). Legendre et Billard (1980) font la même constatation 
chez la truite arc-en-ciel (Salmo gairdneri); ils émettent l'hypothèse d'un vieillissement des spermatozoïdes et proposent d'orienter les études vers l'analyse des constituants ioniques et organiques du liquide sćminal, qui pourraient refléter une certaine aptitude du sperme à la congélation.

D'une manière générale, la composition chimique du liquide séminal des salmonidés est assez bien connue (revue bibliographique de Scott et Baynes, 1980; Piironen et Hyvarinen, 1983; Billard et Menezo, 1984). Cependant peu d'études ont porté sur son évolution au cours de la période de spermiation (Sanchez-Rodriguez et al., 1978; Piironen, 1985; Bayncs et Scott, 1985) et si de nombreux autcurs s'accordent pour penser que la réussite de la congélation dépend en premier lieu de la qualité du sperme, l'absence d'expérimentation sur ce sujet en lait jusqu'à présent un concept abstrait. $A$ ce jour, les expérimentateurs fondent leur choix sur la motilité avant congélation, mais ce critère est apparu très insuffisant (Stoss et Holtz, 1983).

Dans la présente étude nous avons caractérisé chaque sperme testé après congélation, par des critères dont les variations au cours de la spermiation ont été décrites : la concentration en spermatozoïdes, losmolarité, les teneurs en glucose et fructose et le niveau de protéines totales dans le plasma séminal. Par ailleurs, nous nous sommes intéressés à une protéine majeure de la membrane des spermatozoïdes (Loir et Dacheux, 1984, Chambeyron et al., 1987) qui est présente en quantité variable dans le plasma séminal (Loir et Boulard, non publié).

\section{MATÉRIEL ET MÉTHODES}

\section{Les mâles et la collecte du sperme}

Les 40 génitcurs mâles (âge : 3 ans) utilisés, appartenaient à la souche de truite arc-en-ciel "Gournay PR-05-83 " (souche à reproduction printanière). Ils ont ćté marqués individuellement (marque mâchoire numérotée) puis transférés dans un circuit fermé thermo-régulé $\left(12-13^{\circ} \mathrm{C}\right)$ le $21-01-86 . \Lambda$ cette date 13 mâles étaient déjà spermiants, dont 8 en tout début. Les poissons ont été maintenus à jeun jusqu'à fin avril, puis nourris à $0,5 \%$ du poids vif jusqu'à fin juin. Avant chaque prélèvement de sperme les mâles ćtaient anesthésiés dans un bain de 2-phénoxyéthanol $\left(3 \mathrm{~cm}^{3} / 10 \mathrm{dm}^{3}\right.$ d'eau); après la manipulation, ils subissaient un bain éclair de vert malachite ( $70 \mathrm{mg} / 1 \mathrm{dm}^{3}$ pendant 30 secondes) avant d'ĉtre remis dans les bassins d'élévage.

Une collecte hebdomadaire du sperme a été pratiquée à l'aide d'une pipette de transfert à usage unique, qui permet l'aspiration de sperme non contaminé (eau, fécès, urine) dont l'émission est provoquée par pression des flancs de l'animal. En raison du nombre important des analyses pratiquées, seuls les spermes dont le volume était supérieur à $6 \mathrm{~cm}^{3}$ ont été étudiés.
Pendant les manipulations le sperme frais était conservé sur de la glace fondante à l'abri de l'eau et de la lumière.

\section{Les caractérisations physico-chimiques du sperme}

Le nombre de spermatozoïdes par $\mathrm{cm}^{3}$ de sperme a été calculé après dilution au $1 / 2500$ dans de l'eau physiologique $\left(\mathrm{NaCl} 8 \cdot 10^{-3}\right)$ et comptage à l'aide d'une cellule de Thoma.

Les mesures suivantes ont été réalisées sur le plasma séminal obtenu après centrifugation du sperme pendant 20 minutes à $500 \mathrm{~g}$. L'osmolarité a été mesurée à l'aide d'un osmomètre; le glucose et le fructose ont été dosés selon la méthode décrite par Piironen en 1985; les protéines totales ont été dosées par la méthode décrite par Lowry et al. (1951). Enfin, le pourcentage de protéine $42 \mathrm{KD}$ par rapport aux protéines totales du plasma sćminal a été estimé par la méthode suivante : les protéines de chaque plasma séminal considéré ont été analysées par électrophorèse en présence de SDS sur gradient d'acrylamide de 6 à $16 \%$ selon Laemmli (1970). Les gels ont été colorés

Mb A B C D E F G St

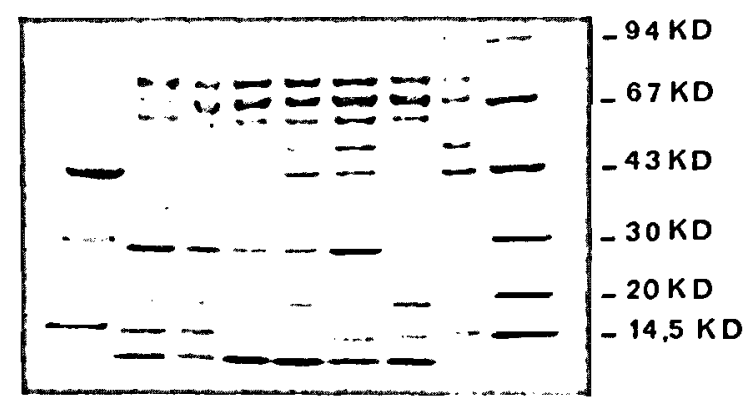

Figure 1. - Gel d'électrophorèse après coloration au bleu de coomassic. $\mathrm{Mb}=$ membranes de spermatozoïdes de truitc are-enciel, préparées selon la technique Jones et al. (1983): A, B, C, D, $E, F, G=$ plasmas séminaux de spermes prélevés le mème jour; $\mathrm{St}=$ standard de poids moléculaires (pharmacia L.MW).

Electrophoresis gel stained with Coomassie blue. $M \mathbf{b}=$ spermatozoan membranes of rainbow trout prepared with the technical described by Jones et al. (1983); $A, B, C, D, E, F, G=$ seminal plasma of milts taken of the same day; $S t=:$ molecular weight standard (pharmacia LMIW).

par le bleu de Coomassic R 250 ( fig. 1) et enregistrés sur un photomètre intégrateur Vernon PHI5. Le pourcentage de protéine $42 \mathrm{KD}$ a été estimé par le rapport de la surface du pic correspondant sur la surface totale de l'électrophorégramme.

Pour chaque poisson, la date d'ćchantillonnage a été définie par le nombre de semaines écoulées depuis le premier prélèvement ayant fourni un volume de sperme significatif (fixé arbitrairement à $2 \mathrm{~cm}^{3}$ ).

\section{La fécondance du sperme frais}

Ce paramètre a ćté ćtudié en procédant à l'insémination d'un mélange d'ovules obtenus à partir de 
femelles (au moins 2) de la même souche que les mâles : $20 \mathrm{~cm}^{3}$ d'ovules ont été fécondés par $0,1 \mathrm{~cm}^{3}$ de sperme dans $10 \mathrm{~cm}^{3}$ de dilueur "DIA 532 " selon la méthode décrite par Billard (1977). L'incubation s'est déroulée dans un circuit fermé thermorégulé à $10^{\circ} \mathrm{C}$. Le taux d'embryonnement à 10 jours a été calculé d'après les comptages réalisés après éclaircissement des oufs dans du liquide de Stockard (5 vol. de formol, 6 vol. de glycérol, 4 vol. d'acide acétique, 85 vol. d'eau distillee).

La congélation et décongélation du sperme, test de fécondation

La congélation du sperme a été effectuéc dans l'heure suivant la collecte. Le dilueur de congélation a ćté cclui mis au point par Mounib (1978) modifić par Legendre et Billard (1980) (sucrose : $125 \mathrm{mM}$, gluthation réduit: $6,5 \mathrm{mM}$, bicarbonate de potassium : $100 \mathrm{mM}$, pH : 7,57, jaune d'œuf au tellurite de potassium : $10 \%$, dimethylsulfoxide : $10 \%$. Le taux de dilution du sperme a été de 1 volume de sperme pour 2 volumes de dilueur. Le mélange a été conservé sous forme de boulettes de $0,1 \mathrm{~cm}^{3}$, congelées au contact de carboglace selon la technique de Nagaze (1964) puis maintenues dans de l'azote liquide jusqu'à la décongélation.

La technique de décongélation et d'insémination s'inspire de celle préconisée par Legendre et Billard (1980) : 3 boulettes sont plongées directement dans $5 \mathrm{~cm}^{3}$ de «DIA 532 » à $25^{\circ} \mathrm{C}$. A près 10 secondes, ce mélange est ajouté à $20 \mathrm{~cm}^{3}$ d'ovules et $5 \mathrm{~cm}^{3}$ de « DIA 532 ». La suite de la manipulation est la même que pour l'insémination avec du sperme frais. La décongélation et l'insémination ont été pratiquées le 02-12-1986 soit 5 à 9 mois après congélation. Les ovules provenaient de femelles (âge 3 ans) de la souche " Gournay SY-01-83 ". Le taux d'embryonnement a été calculé après 10 jours d'incubation à $10^{\circ} \mathrm{C}$ comme ci-dessus.

\section{Analyses statistiques}

Les corrèlations entre la fécondance du sperme congelé et ses caractéristiques physico-chimiques ont été étudiées après réalisation d'un tableau de contingence croisant les modalités de la variable "taux d'embryonnement " avec les modalités des variables "caractéristiques physico-chimiques". Nous avons ensuite procédé à une analyse factorielle des correspondances sur le tableau de contingence ainsi créé (Benzecri, 1980; Bachacou et al., 1981).

\section{RESULTATS}

La fécondance du sperme frais varie suivant les mâles et les lots d'ovules inséminés (fig. 2). Avec le sperme congelé les taux d'embryonnement à 100 degrés-jours se distribuent en deux groupes: de
0 à $35 \%$ et de 40 à $100 \%$ (fig. 2). 11 n'existe pas de corrélation entre la fécondance à l'état frais et celle après congélation. De mème il n'y a pas de correspondance entre les performances relatives des spermes frais (tenant compte d'un probable effet « qualité des ovules $")$ et celles après congélation.
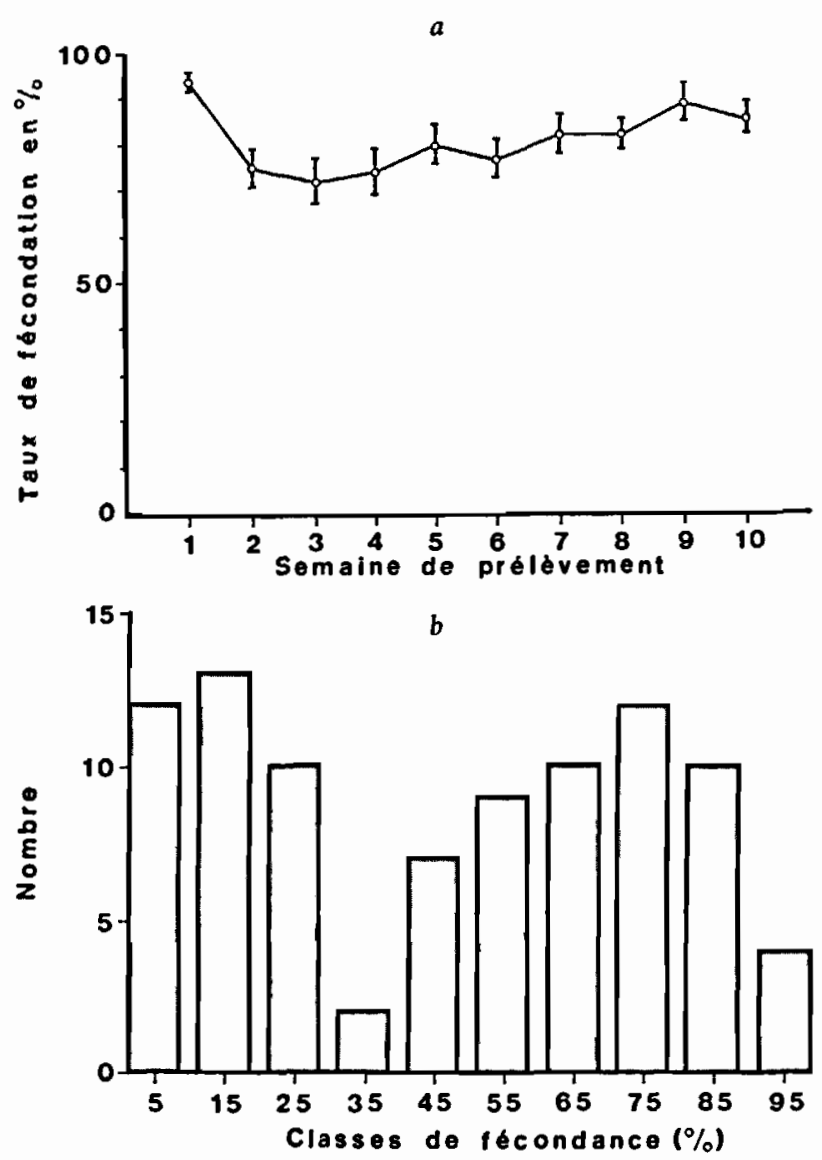

Figure 2. $-a-$ Fécondance du sperme frais $(n=8$, moyenne, éart (ype). $b$ - Distribution des classes de fécondance après cryoconservation. (nombre total de spermes : 91 ).

$a$ - Fertility of fresh milt ( $n=8$, acerage standard Error). $b-$ Distribution of fertility classes after cryopresertation (total number of milts: 91).

Les 91 spermes ont été prélevés sur 26 mâles, cependant seuls 5 mâles ont permis un suivi individuel des différents paramètres pendant au moins 5 semaines ( $f i g .3$ ). Les niveaux et les évolutions des paramètres considérés montrent de grandes différences entre les mâles et des variations importantes au cours de la période. Le volume hebdomadaire de sperme collecté double pendant les 6 premières semaines. La deuxième partie de la période de spermiation se caractérise par de grandes variations individuclles. Dans ccrtains cas le volume reste constant chaque semaine puis chute. Pour d'autres, il $y$ a une élévation brutale la $8^{\circ}$ semaine avec un doublement du volume collecté et des fluctuations importantes d'une semaine à l'autre. 

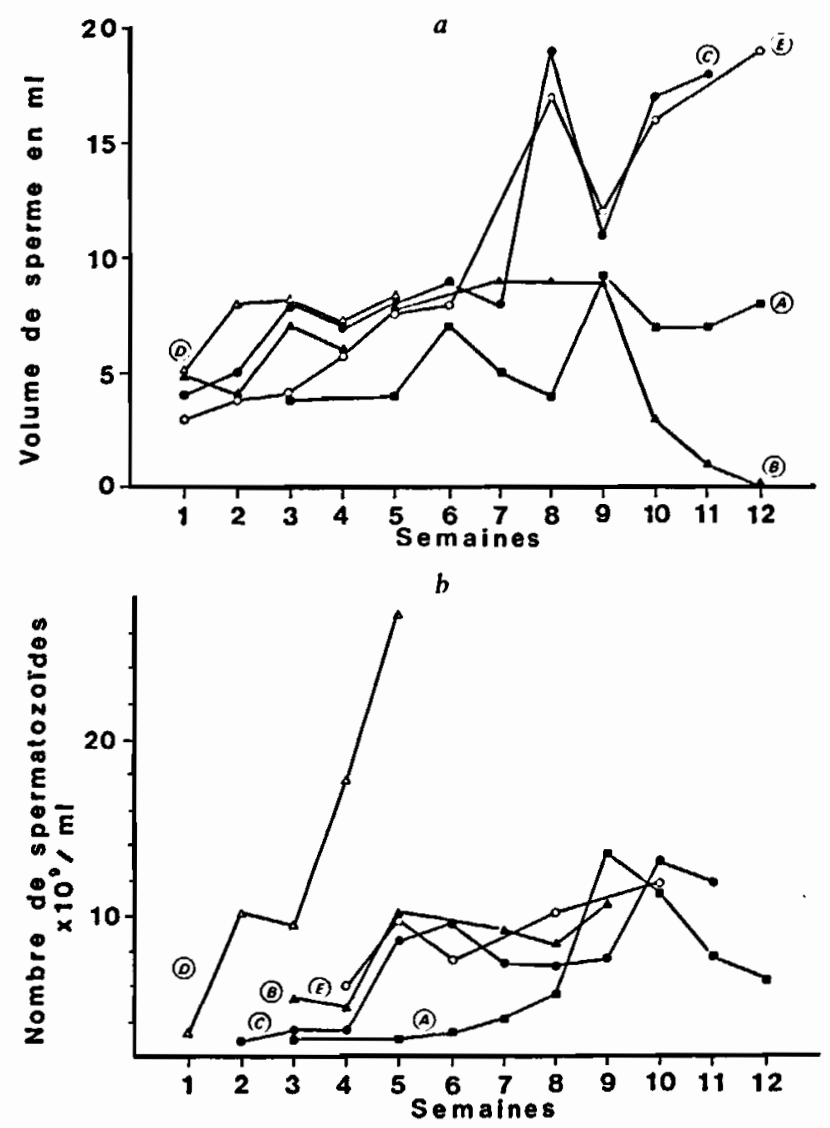

Figure 3. - Evolutions individuelles du volume de sperme (a) et de sa concentration en spermatozoïdes $(b)$.

Individual evolutions of milt volume $(a)$ and spermatozoan concentration (b).

La concentration du sperme en spermatozoïdes varie de manière importante suivant les poissons et les dates de collecte. Au début de la période de spermiation le sperme est peu concentré, puis le nombre de spermatozoïdes par $\mathrm{cm}^{3}$ augmente plus ou moins rapidement et régulièrement selon les individus.

L'étude des plasmas séminaux montre que de la même façon des différences cntre mâles et suivant la date d'échantillonnage ( fig. 4). Il est à noter que l'évolution dans le temps de l'" osmolarité ", de la "tencur en protéines" et du «pourcentage de la protéine $42 \mathrm{KD}$ ", pour chacun des 5 poissons, suggère l'existence possible de variations parallèles de ces 3 paramètres. Le glucose et le fructose n'ont ćté trouvés qu'à l'ćtat de traces pour l'ensemble des 91 spermes étudiés.

L'incidence éventuelle des caractéristiques physicohimiques du sperme sur la fécondance après congélation a été abordée par l'analyse factorielle des correspondances dans le tableau de contingence réalisé (les différentes modalités ont été définies à partir de l'étude de la distribution des variables).
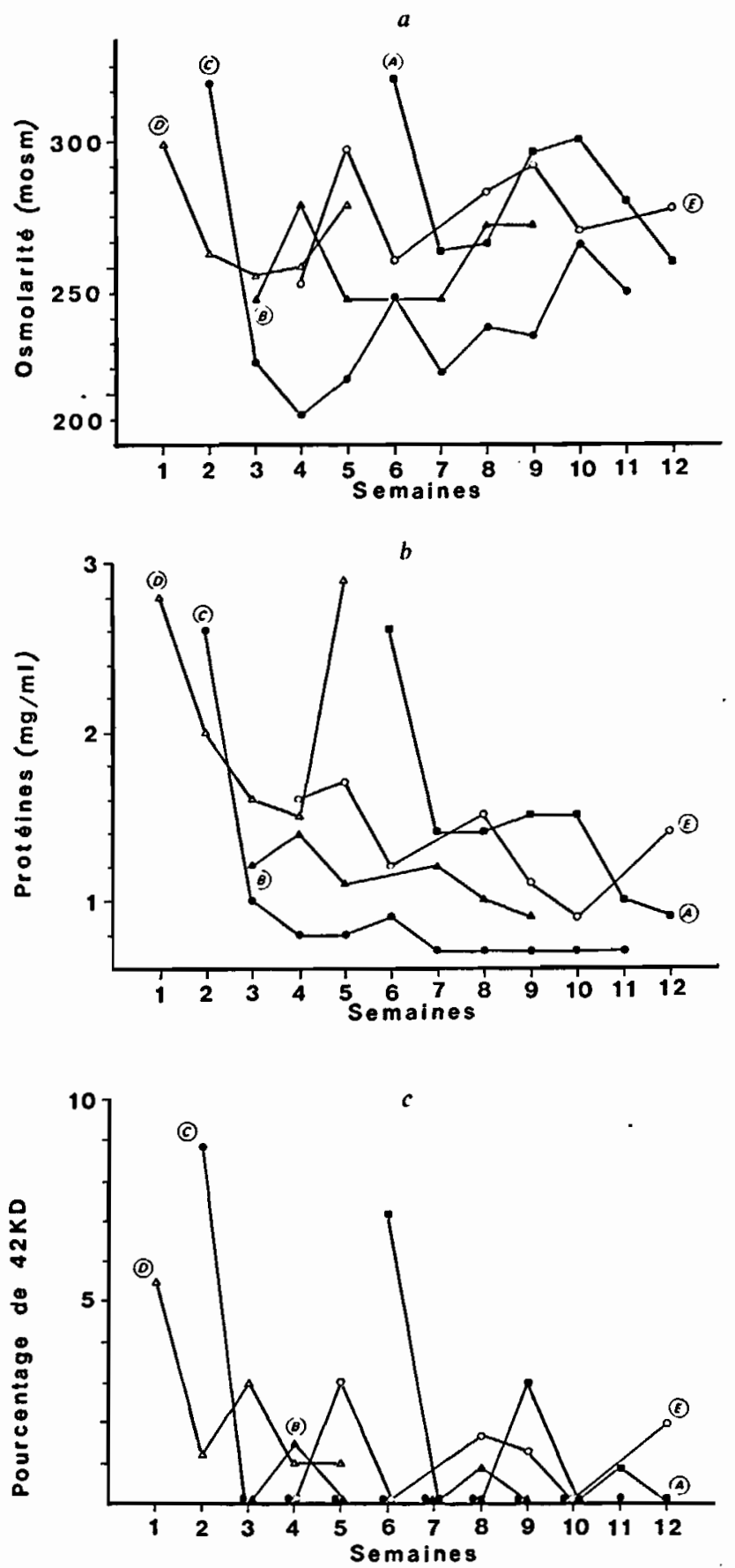

Figure 4. - Évolutions individuelles des caractéristiques du plasma séminal : $a$ - Osmolarité. $b-$ Teneur en protéines totales. $c-$ Pourcentage en protéines $42 \mathrm{KD}$.

Inditidual etolutions of characteristics of seminal plasma: a - Osmolality. $b$ - Total proteins level. $c-42 K D$ protein percentage.

En lignes: la fécondance ( $F$ en $\%$ du sperme congelé avec 5 modalités : FEC-1 $(0 \leqq \mathrm{~F}<20)$; FEC$2 \quad(20 \leqq F<40) ; \quad F E C-3 \quad(40 \leqq F<60) ; \quad F E C-4$ $(60 \leqq F<80) ; F E C-5(80 \leqq F \leqq 100)$.

En colonnes : 
- la teneur en protéines totales ( $\mathrm{T}$ en $\mathrm{mg} / \mathrm{cm}^{3}$ )

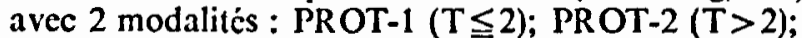

- la teneur en protéine $42 \mathrm{KD}$ ( $P$ en $\%$ de $T$ ) avec 5 modalités: $\% 42 \mathrm{KD}-1 \quad(\mathrm{P}=0) ; \% 42 \mathrm{KD}-2$ $(0<\mathrm{P} \leqq 1,5) ; \% 42 \mathrm{KD}-3 \quad(1,5<\mathrm{P} \leqq 2,5) ; \% 42 \mathrm{KD}-4$ $(2,5<\mathrm{P} \leqq 4,0) ; \% 42 \mathrm{KD}-5(\mathrm{P}>4,0)$;

- l'osmolarité (PO en mosmoles) avec 3 modalités : OSMO-1 (PO $\leqq 220) ; \quad$ OSMO-2 $(220<\mathrm{PO} \leqq 260)$; OSMO-3 (PO>260);

- la concentration $\left(\mathrm{N} \times 10^{\circ} / \mathrm{cm}^{3}\right)$ du sperme en spermatozoïdes avec 6 modalités : NBSPZ-0 (données manquantcs): NBSPZ-1 ( $\mathrm{N} \leqq 6) ; \quad$ NBSPZ-2 $(6<N \leqq 10) ; \quad N B S P Z-3 \quad(10<N \leqq 14) ; \quad N B S P Z-4$ $(14<N \leqq 18) ; N B S P Z-5(N>18)$.

La représentation graphique des résultats de l'analyse factoriclle des correspondances pratiquée sur cc

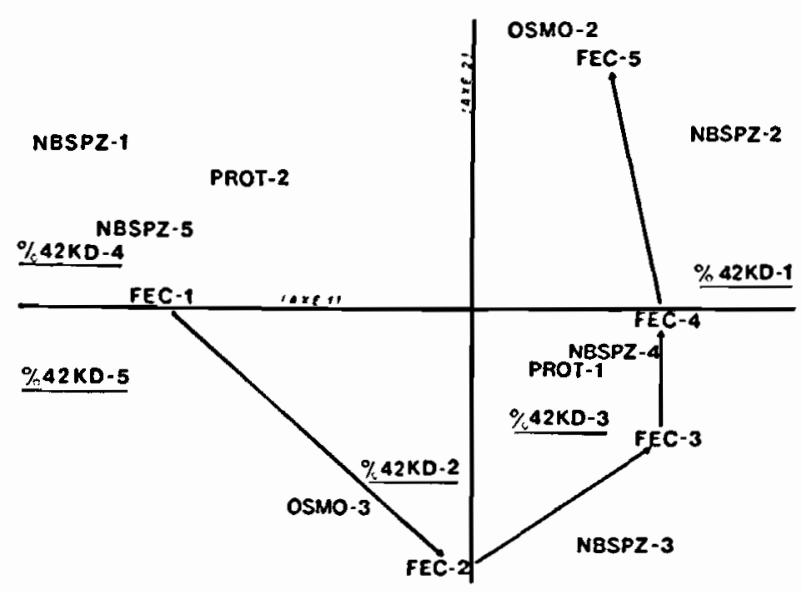

Figure 5. - Relations entre la fécondance du sperme congelé et ses caractéristiques physico-chimiques à l'état frais: le plan des deux premicrs axes factoricls issus de lanalyse du tableau de contingence (tabl. 1).

Relationships between frozen milt fertility and physico-chemical characteristics of fresh sperm: the two first factorial axis from the analysis of the contingency table (tabl. 1). tableau est donnée dans la figure 5. Les pourcentages d'inertie des axes 1 et 2 sont respectivement 56 et $27 \%$.

Compte tenu des qualités de représentation de chaque point il ressort que l'axe 1 oppose les spermes à fécondance très médiocre (FEC-1) à ceux pour lesquels les résultats sont moyens (FEC-3) et bons (FEC4). Les premiers sont associés à des teneurs en protćines totales élevées (PROT-2), des teneurs en protéine $42 \mathrm{KD}$ importantes (\%42 KD-4; \%42 KD-5) et des concentrations en spermatozoïdes très raibles (NBSPZ-1) ou très fortes (NBSPZ-5). Les seconds sont associés à des teneurs en protéines totales modérées (PROT-1), et à l'absence de protéine $42 \mathrm{KD}$ $(\% 42 \mathrm{KD}-1)$.

L'axe 2 oppose les spermes à fécondance médiocre (FEC-2) aux très bons spermes (FEC-5). Les premiers sont associés à des osmolarités élevées (OSMO-3) et les seconds à des osmolarités moyennes (OSMO-2).

\section{DISCUSSION}

Les résultats des fécondations réalisées avec du sperme frais indiquent que nous avons toujours travaillé avec du sperme de bonne qualité. L'absence de correspondance entre les performances à l'état frais ct après congélation doit se situer dans ce cadre, car il est probable qu'un sperme frais médiocre restera médiocre après congélation.

La production totale en spermatozoïdes variant de $360 \times 10^{9}$ à $950 \times 10^{9}$ par mâle est comparable à celles obscrvécs par Buyukhatipoglu et Holtz (1984). La figure 6 qui présente l'évolution du nombre cumulé de spermatozoïdes par mâle en fonction de la date d'échantillonnage suggère l'existence de plusieurs stratćgics : une production rapide se caractérisant par une augmentation importante et précoce de la concentration en spermatozoïdes (mâle D); unc production régulière plus ćtalće avec des variations importantes du volume des éjaculats dont la concentration en

Tableau 1. - Le tableau de contingence, croisant les modalités de la variable « Fécondance " avec les modalités des variables " caractéristiques physico-chimiques ", sur lequel il a été procédé à l'analyse factorielle des correspondances.

The contingency table crossing modalities of the tariable "Fertility" and modalities of the rariables "physico-chemical characteristics", on which factorial analy sis of correspondences nas performed.

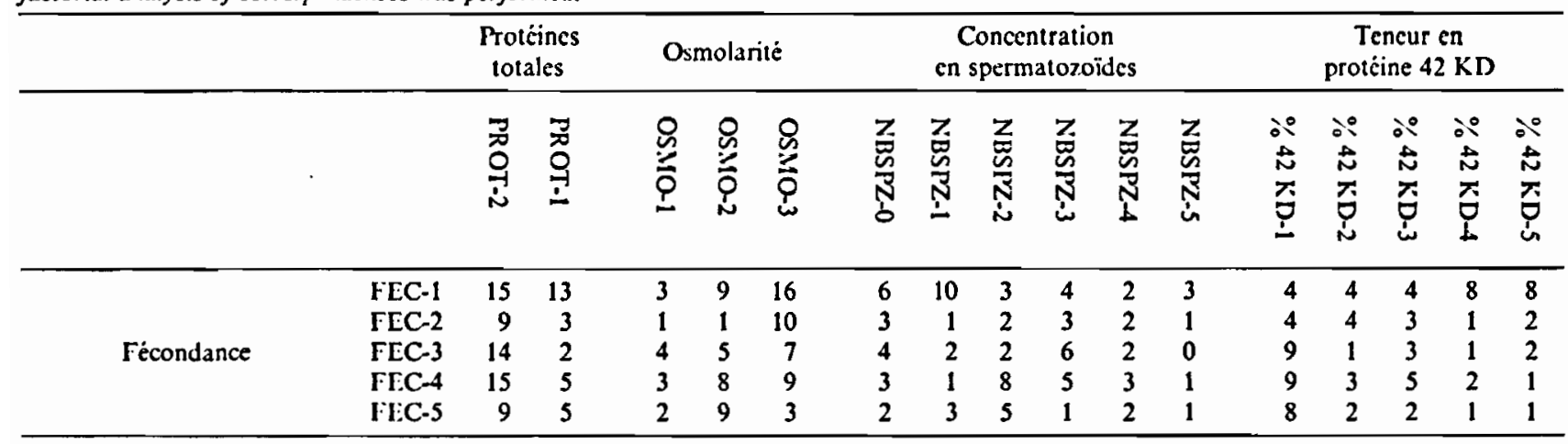




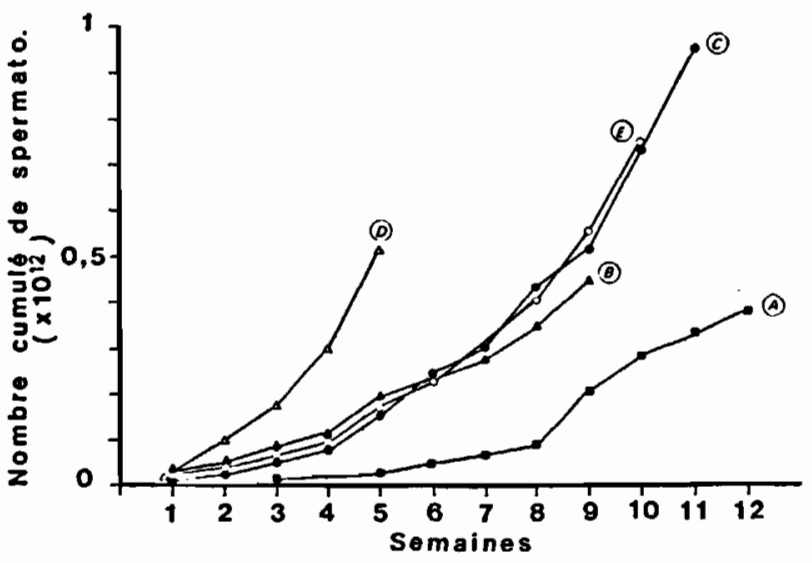

Figure 6. - Les différentes stratégies de spermiation: nombre cumulé de spermatozoides collectés.

The various spermiation strategies: cumulatice number of spermatozoa.

spermatozoïdes, bien que variable, reste moyenne (mâles B, C et E); une production plus lente à se mettre en place caractérisée principalement par une densité faible jusqu'à la $8^{e}$ semaine (mâle $\mathrm{A}$ ).

Les valeurs de l'osmolarité, bien que très dispersées puisqu'elles sont comprises entre 174 et 364 mosmols, correspondent en moyenne à celle donnée par Morisawa (1985) pour la truite arc-en-cicl. Les valeurs observées pour la teneur en protéines totales sont voisines de celles trouvées par Sanchez-Rodriguez et al. (1978). La corrélation notéc pour chaque individu entre l'osmolarité et la teneur en protéines totales va dans le sens d'un phénomène de dilution du plasma séminal. Il faut noter cependant que des variations importantes de l'osmolarité sont contemporaines de modifications de la teneur en protéine $42 \mathrm{KD}$. La présence dans le plasma séminal de cette protéine peut être un indicateur de la dégradation de la membrane des spermatozoïdes, dont elle est un des principaux constituants (Loir et Dacheux, 1984). Il est possible que cette dégradation s'accompagne d'une libération dans le liquide séminal d'acides aminés et d'ions qui agiraient sur l'osmolarité. Il n'est cependant pas exclu que ce soient les variations de l'osmolarité qui agissent sur l'intégrité de la membrane des spermatozoides, et qui soient à l'origine de la présence de la protéine $42 \mathrm{KD}$ dans le liquide séminal.

L'analyse des performances de chaque sperme congelé en relation avec ses caractéristiques physicochimiques va dans le sens d'une influence de la qualité de la membrane, hypothèse déjà formuléc par Baynes et Scott (1982) et Yoo et al. (1987). En effet, ce sont les spermes dont le plasma séminal a une forte tencur en protéine $42 \mathrm{KD}$ et/ou une forte osmolarité qui supportent le moins bien la congélation.

L'influence négative des faibles concentrations en spermatozoïdes peut s'expliquer par un nombre insuffisant de spermatozoïdes par ovule au moment de l'insémination. En effet, la modalité "NBSPZ-1 " correspond à un nombre de spermatozoïdes par ovule inféricur à $3 \times 10^{6}$ qui est la valeur minimale donnée par Stoss et Holtz (1981). Il est cependant paradoxal de constater que les très fortes concentrations sont aussi associées à de mauvais résultats. Il faut préciser que dans ce cas nous sommes en présence de mâles dont la spermiation est très courte (cas du mâle D).

L'absence de variations des taux de glucose et de fructose nous permettent d'affirmer que les variations de qualité des spermes congelés que nous avons testés ne peuvent pas être correlées à ces paramètres, sans préjuger cependant de l'éventuelle influence de fluctuations comparables à celles décrites par Piironen (1985) chez le Saumon finlandais.

D'autres protéines du plasma séminal (29 KD, $19 \mathrm{KD}, 15 \mathrm{KD}, 11 \mathrm{KD}$ ) varient suivant les spermes ( fig. 11) sans que l'on ait pu mettre en évidence de relation avec l'aptitude à la cryoconservation (analyse factorielle des correspondances).

\section{CONCLUSION}

Cette étude a permis de mettre en évidence un certain nombre de relations entre l'aptitude du sperme à la cryoconservation et certaines de ses caractéristiques physicochimiques. La teneur en protéine $42 \mathrm{KD}$ est apparue comme le critère étudié le plus discriminant. La présence de cette protéine membranaire dans le plasma séminal suggère une certaine fragilité de la membrane des spermatozoïdes, la rendant inapte à la congélation-décongélation. Chez le saumon atlantique, une protéine d'un poids moléculaire voisin, $45 \mathrm{KD}$, peut totalement disparaître de la membrane des spermatozoïdes après cryoconservation, pour ne se retrouver qu'au niveau du plasma séminal (Yoo et al., 1987). Il semble donc bien que la liaison de cette protéine aux autres constituants de la membrane soit suffisamment fragile pour que toute dégradation membranaire se traduise par une augmentation importante de sa tencur dans le plasma séminal. Nos résultats restent cependant insuffisants pour une application pratique et une approche plus précise de la composition du plasma séminal et de la membrane des spermatozoîdes apparaît nécessaire.

\section{REFERENCES}

Bachacou J., J. P. Masson, C. Millier, 1981. Manuel de la programmathèque statistique AMANCE 81. Département de biométrie. INRA, Copedith, $516 \mathrm{p}$.

Baynes S. M., A. P. Scott, 1982. Cryoprescrvation of rainbow trout spermatozoa : variation in membrane composition may influence spermatozoan survival. In: Reproductive physiology of fish. Richter C. J. J., H. S. Th. Goos Ed., PUDOC, Wageningen. 
Baynes S. M., A. P. Scott, 1985. Seasonal variations in parameters of milt production and in plasma concentration of sex steroids of male rainbow trout (Salmo gairdneri). Gen. Comp. Endocrinol., 57, 150-160.

Benzecri J. P., 1980. L'analyse des données; tome 1 : La Taxinomie; tome 2: L'Analyse des correspondances, Dunod, Paris, 2 vol., 625 p +632 p.

Billard R., 1977. Utilisation d'un système tris-glycocolle pour tamponner le dilueur d'insémination pour truite. Bull. Fr. Pisc., 264, 102-112.

Billard R., Y. Menezo, 1984. The amino acid composition of rainbow trout (Salmo gairdneri) seminal fluid and blood plasma: a comparison with carp (Cyprinus carpio). Aquaculture, 41, 255-258.

Buyukhatipoglu S., W. Holtz, 1984. Sperm output in rainbow trout (Salmo gairdneri). Effect of age, timing and frequency of stripping and presence of females. Aquaculture, 37, 63-71.

Chambeyron F., M. Loir, B. Mourot, 1987. Caractérisation des protéines du Muide testiculaire et du plasma séminal de truite arc-en-ciel (Salmo gairdneri). $2^{\mathbf{e}}$ Réunion Française de Physiologie des Poissons, Bordeaux 7-10 septembre $1987,120 \mathrm{p}$.

Jones R., K. I. Von Gloss, C. R. Brown, 1983. Changes in the protein composition of rat spermatozoa during maturation in the epididymis. J. Reprod. Fert., 67, 299306.

Laemmli U. K., 1970. Cleavage of structural proteins during the assembly of the head of bacteriophage T4. Nature, 227, 680-681.

Legendre M., R. Billard, 1980. Cryopreservation of rainbow trout sperm by deep-freczing. Reprod. Nutr. Detelop., 20, 1859-1868.

Loir M., J. L. Dacheux, 1984. Inventaire électrophorétique des protéines du fluide testiculaire, du plasma séminal, des membranes et des flagelles des spermatozoïdes de truite. Réunion "physiologie des poissons ", INRAIFR EMER, Paimpont 1-3 octobre 1984, 48 p.

Lowry O. H., N. J. Rosebrough, A. L. Farr, R. J. Randall, 1951. Protein measurement with the Folin phénol reagent. J. Biol. Chem., 193, 265-275.

Mounib M. S., 1978. Cryogenic preservation of fish and mammalian spermatozoa. J. Reprod. Fert., 53, 13-18.
Morisawa M., 1985. Initiation mechanism of sperm motility at spawning in teleosts. Zool. Sci., 2, 605-615.

Nagase H., 1964. Deep freezing bull semen in concentrated pellet form. I. Factors affecting survival of spermatozoa. Proc. Int. Congr. Reprod. Anim. Insem. Artif., Trento, 4, 410-415.

Ott A. G., 11. F. Horton, 1971. Fertilization of chinook and coho salmon eggs with cryo-preserved sperm. $J$. Fish. Res. Bd. Canada, 28, 745-748.

Piironen J., 1985. Variation in the properties of milt from the finnish landlocked salmon (Salmo salar m. sebago Girard) during a spawning scason. Aquaculture, 48, 337350.

Piironen J., H. Hyvarinen, 1983. Composition of the milt of some telcost fishes. J. Fish. Biol., 22, 351-361.

Sanchez-Rodriguez M., A. M. Escafrre, S. Marlot, P. Reinaud, 1978. The spermiation period in the rainbow trout (Salmo gairdneri). Plasma gonadotropin and androgen levels, sperm production and biochemical changes in the seminal fluid. Ann. Biol. Anim. Bioch. Biophys., 18, 943-948.

Scott A. P., S. M. Baynes, 1980. A review of the biology, handling and storage of salmonid spermatozoa. J. Fish Biol., 17, 707-739.

Stoss J., 1983. Fish gamete preservation and spermatozoan physiology. In: Fish physiology, Hoar W. S., D. J. Randall, E. M. Donaldson Ed., vol. IX, Reproduction, part B, Behavior and fertility control. Academic Press, New York, 305-350.

Stoss J., W. Holtz, 1981. Cryopreservation of rainbow trout (Salmo gairdneri) sperm. I. Effect of thawing solution, sperm density and interval between thawing and insemination. Aquaculture, 22, 97-104.

Stoss J., E. M. Donaldson, 1982. Preservation of fish gametes. In: Reproductive Physiology of fish, Ritcher C. J. J., H. S. Th. Goos, Ed., PUDOC, Wageningen, 114-122.

Stoss J., W. Holtz, 1983. Cryopreservation of rainbow trout (Salmo gairdneri) sperm. III. Effect of proteins in the diluent, sperm from different males and interval between sperm collection and freezing. Aquaculture, 31, 275-282.

Yoo B. Y., M. A. Ryan, A. J. Wiggs, 1987. Loss of protcin from spermatozoa of Atlantic salmon (Salmo Salar L.) because of cryopreservation. Can. J. Zool., 65, 9-13. 